\title{
Refíïg'z
}

\section{ausarmählte $\mathfrak{W}_{\mathfrak{e r k e}}$.}

F̧ünter Band.

Stuttgart.

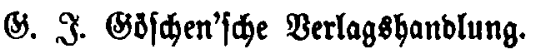

1873. 
Drut von Rart eItn in Etultgart. 